\title{
High Connectivity with Minimum BER in Wireless Sensor Networks
}

\author{
Mohammed Amal \\ Computer Engineering Department \\ Faculty of Engineering \\ Zagazig University, cairo, Egypt
}

\author{
Ben Bella S. Tawfik \\ Faculty of Computers \& Informatics \\ Suez Canal University \\ Ismailila, Egypt
}

\begin{abstract}
Power saving is considered a significant issue relating to wireless ad hoc and sensor networks, where nodes normally use limited battery power. Transmitting using unnecessary high power introduces excessive interference. It is much better if the network designer would have each node transmit at the lowest possible power while the network connectivity is preserved.

This paper introduces the optimal common transmit power, defined as the minimum transmit power used by all nodes necessary to guarantee network connectivity. This is desirable in sensor networks since nodes are relatively simple, while modifying transmit power after deployment is difficult.

The optimal transmit power used in this paper has been derived in previous work. It is subject to the specific routing and medium access control (MAC) protocols considered. However, the approach can be additionally extended to other routing and MAC protocols. In this paper, connectivity is defined in terms of a quality of service (QoS) constraint given by the maximum tolerable bit error rate (BER) at the end of a multihop route with an average number of hops.
\end{abstract}

\section{General Terms}

Sensor networks, Ad hoc wireless networks, power saving, network connectivity.

\section{Keywords}

medium access control (MAC), quality of service (QoS), bit error rate (BER), probability mass function (pmf).

\section{INTRODUCTION}

Power conservation is an important issue in an ad hoc wireless network, where nodes are likely to operate on limited battery life. Conserving power prolongs the lifetime of both the node and the network. In addition, transmitting at low power reduces the amount of excessive interference. Thus, the question arises primarily should be: "What is the optimal transmit power that should be used?" This paper tries to answer this question. Obviously, a suitable criterion of optimality has to be introduced.

Having a network connectivity is amongst the goals of forming a network. This is accomplished when each node is able to communicate with any other nodes, possibly via multiple hops. The connectivity level of an ad hoc wireless network depends on the transmit power of the nodes. If the transmit power is too small, the network might get disconnected. However, as menti transmitting at excessively high power is ineffective due to mutual interference in the shared radio channel and the limited battery lifetime, it is clear that the optimal transmit power is the minimum power enough to grant network connectivity [1], [2], [3].

Ideally, to achieve the best optimal power the transmit power of a node should be adjusted on a link-by-link basis [2], [3], [4], [5], [6]. And since performing power control on a link bylink basis is a complicated burdensome task, a simpler more viable solution for implementation is to have all the nodes use a common transmit power. This is desirable in sensor networks, where nodes are relatively simple, and after deployment, it is difficult to modify the transmit power. Moreover, the performance difference between adjusting the power locally and employing a common transmit power is small, especially when the nodes number is large [1]. This paper investigates the optimal transmit power for an ad hoc wireless networking scenario, where all nodes use a common transmit power.

It is noteworthy that although there may be a path connecting two nodes, communication between them may not be possible as the QoS in terms of tolerable BER at the end of a multihop route may not be satisfied. Thus, this paper discusses the BER in more detail, where the optimal transmit power sufficient to maintain network connectivity is found according to a physical layer-oriented QoS constraint given by the maximum tolerable BER at the end of a multihop route with an average number of hops.

Analytically, the paper evaluates the optimal transmit power, and investigates the interrelation among optimal transmit power, data rate, and node spatial density. The paper also investigates 1) The impact of different propagation pathloss exponents (on different links of a multihop route) on the performance of a common transmit power control scheme.2) The interrelation among transmit power, connectivity, and network longevity. Furthermore, this paper provides a thorough analysis of exact interference power and BER, using a detection theory approach as opposed to simply assuming the interference on each link is the average power as considered in [10]. Then, a validation on analysis with simulation is implemented. The remainder of this paper is organized as follows: Section 2 defines the model and assumptions used to derive the optimal transmit power. Section 3 explains network connectivity. Section 4 analyzes the optimal common transmitted power sufficient to maintain network connectivity in both regular and random topologies. The same section includes results and analysis, while section 5 delivers the conclusion. 


\section{MODEL AND ASSUMPTION}

This section defines the basic ad hoc wireless network communication model, and basic assumptions considered in this paper.

\subsection{Network Topology}

Throughout the paper, a scenario is assumed where $\mathrm{N}$ nodes are distributed over a surface with finite area A. The node spatial density is defined as the number of nodes per unit area

$2 R$

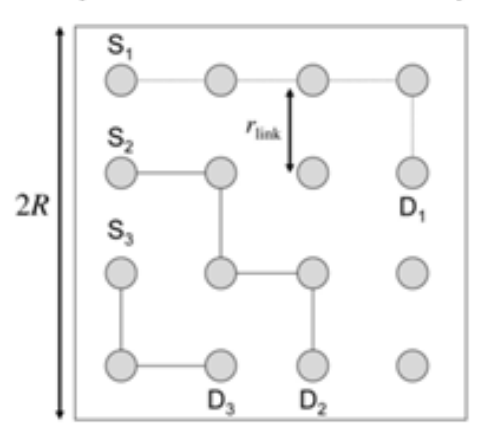

(a) and is denoted as $\rho_{-} s$. To avoid edge effects, we assume the network surface to be the surface of a torus with length $2 \mathrm{R}$ on each edge, as shown in Fig. 1. In a real scenario, the performance predicted by this analysis may not be particularly precise for nodes on the edge of the network surface. In this case, a more precise performance evaluation may be obtained via simulations. Nonetheless, the results presented in this paper provide a representative description of a realistic network behavior.

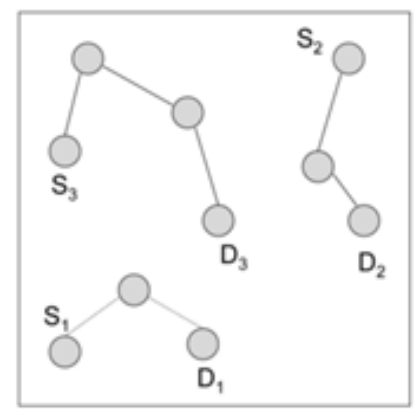

(b)

Fig 1: Possible topologies: (a) regular (b) random

The paper also considers a realistic scenario with twodimensional Poisson node distribution. In a network with square grid topology, as shown in Fig. 1 (a), each node has four nearest neighbors at a fixed distance. In contrast, the positions of nodes in a network with a two-dimensional

$$
\operatorname{Pr}\left(N_{a}=j\right)=\frac{\left(\rho_{s} a\right)^{j}}{j !} e^{-\rho_{s} a}
$$

Where, in this case, $\rho$ s corresponds to the average number of nodes per unit area or the average node spatial density. Considering the same value of $\rho \_s$ for both types of networks with regular and random topologies makes the comparison between them fair and meaningful. This paper considers only ad hoc wireless networks with stationary nodes.

\subsection{Routing}

2.3 An assumption is made of a simple routing strategy; a packet is relayed hop-by-hop, through a sequence of nearest neighboring nodes until it reaches the destination. Another assumption is made that a source node discovers a route prior to data transmission [14]. Discovery of a multihop route from
Poisson topology are random and independent of one another, as shown in Fig. 1 (b). The probability mass function (pmf) of the number of nodes Na over a surface of area a in the case with two-dimensional Poisson topology is given by:

$j=0,1,2, \ldots$,

a source to a destination is a crucial phase in a wireless networking scenario with flat architecture. However, this paper focuses on the characterization of the steady state behavior of on-going peer-to-peer multihop communications. Therefore, it is assumed that there is a route between source and destination. The next section discusses routing in networks with regular and random topologies.

\subsubsection{Square Grid Topology}

2.3.2 Due to the regularity of this topology, the distance to the nearest neighbor, denoted by rlink, is fixed, and a route is constituted by a sequence of hops with equal length.

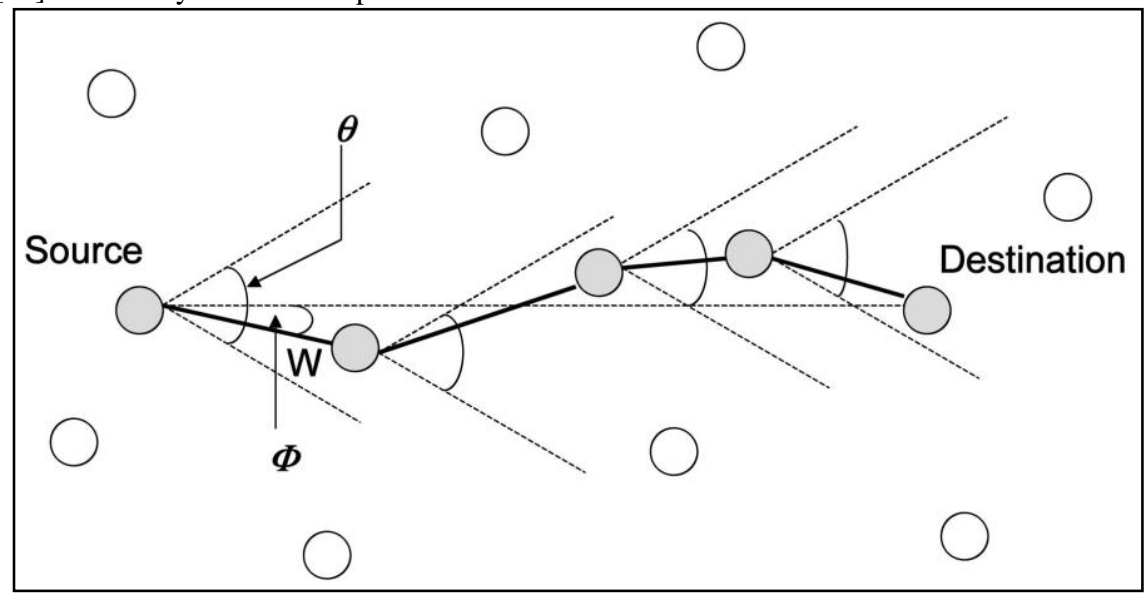

Fig 2:Possible multihop route in a random topology. W corresponds to the distance to the nearest neighbor, $\phi$ corresponds to the projection angle, and $\theta$ corresponds to the angle which a node looks for a neighbor in the destination direction 
The distance rlink can be computed as follows: One can first observe that constructing a square lattice of $\mathrm{N}$ nodes over a surface of a torus with area $\mathrm{A}$ is equivalent to fitting $\mathrm{N}$ small square tiles of area $\mathrm{r} 2$ link into a large square of area $\mathrm{A}$.

The distance to the nearest neighbor can be written as:

$$
r_{\text {link }}=\sqrt{\frac{A}{N}}=\frac{1}{\sqrt{\rho_{s}}}
$$

\subsubsection{Two-Dimensional Poisson Topology}

In the case of random topology, a routing scheme is considered, where each intermediate node in a multihop route relays the packets to its nearest neighbor in the destination direction. Particularly, it is assumed that an intermediate node in the route selects the nearest node within a sector of angle toward the direction of the destination as the next hop [15]. Fig. 2 shows an example of a multihop route constructed in this way. In this case, a route can be visualized as a deviation from the straight line between source and destination, referred to as reference path. Unlike the grid topology scenario, in a network with two-dimensional Poisson topology, the distance from a node to its nearest neighbor is not a constant. Let $\mathrm{W}$ be a random variable denoting the distance to the nearest neighbor in a two dimensional Poisson node distribution. It can be shown that, keeping the node spatial density fixed, for large $\mathrm{N}$, the CDF of the distance to the nearest neighbor in a torus is:

$$
\begin{aligned}
& F_{W}(w) \\
& = \begin{cases}0 & w<0 \\
1-e^{-\rho_{s} \pi w^{2}} & 0 \leq w<R \\
1-e^{-\rho_{s} \chi} & R \leq w<\sqrt{2} R \\
1 & w \geq \sqrt{2} R\end{cases}
\end{aligned}
$$

It can be rewritten as:

$$
F_{w}^{(\theta)}(w)= \begin{cases}1 & w>R \\ 1-e^{-\rho_{s} \frac{\theta w^{2}}{2}} & 0 \leq w \leq R \\ 0 & \text { otherwise }\end{cases}
$$

More details on the derivation can be found in [16]. In this paper, a consideration of a simple reservation-based MAC protocol introduced in [17] and defined as reserve-and-go (RESGO).1 In this protocol, a source node first reserves intermediate nodes on a route for relaying its packets to the destination-characterization of this phase is beyond the scope of this paper. A transmission can begin after a route is discovered and reserved. The main idea of the protocol is that a source node or a relay node generates an exponential random backoff time before it transmits or relays each packet. After the random backoff time expires, a node can start transmitting a packet. The random backoff time helps reduce interference among nodes in the same route as well as in different routes. Throughout this paper, it is assumed that the random backoff time is exponential with mean $1=$ t. In other words, given that a node has packets to send, packets are transmitted with rate $\lambda \mathrm{t}$ (dimension: [pck/s]). Note that this is generally different from the traffic generation rate, $\lambda \mathrm{g}$.

\section{CONNECTIVITY}

As discussed earlier, the optimal common transmit power is the minimum power sufficient to preserve network connectivity. In this section, the definition of network connectivity is formalized. Conceptually, an ad hoc wireless network is often viewed as a graph, where vertices represent the nodes and edges represent the links connecting neighboring nodes. From a graph-theoretical perspective, a network is connected if there is a path (possibly multihop) connecting any node to any other node in the network. Nevertheless, it can be misleading to use this connectivity notion for an ad hoc wireless network, where a communication channel is subject to error. Since the wireless links are prone to errors, the QoS in terms of route BER declines while the number of hops in a route increases. Thus, the performance may be unacceptable even though there is a links sequence to destination. This paper considers the network connectivity from a communication-theoretic viewpoint in order to bear the physical layer characteristics in mind. In particular, a network can be seen as connected when any source node can communicate with a BER lower than a prescribed value BERth to a destination node placed at theend of a multihop.

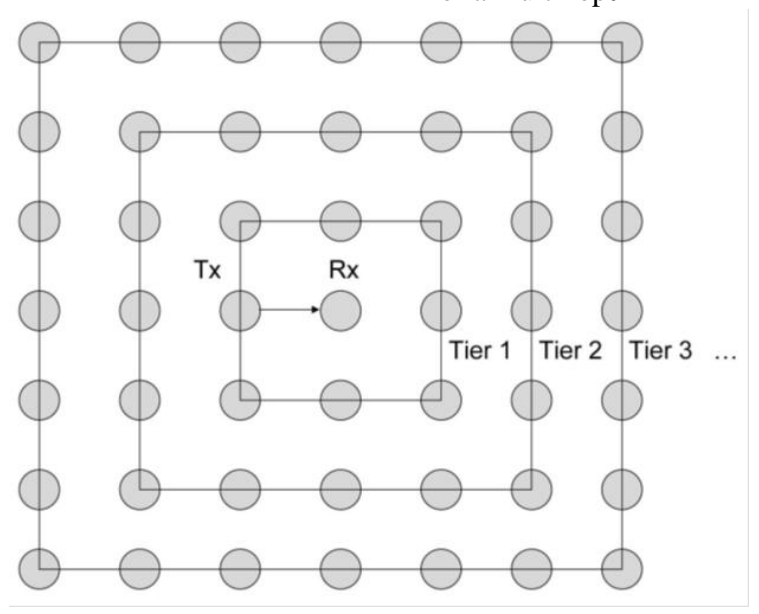

Fig 3:Tier structure of a network with square grid topology

Evidently, the received signal observed at the receiver is the sum of three components: 1) Intended transmitter signal, 2) Interfering signals from other nodes, and 3) Thermal noise. Since the interfering signals come from other nodes, it is assumed that the total interfering signals can be treated as an additive noise process independent of the thermal noise process. The received signal $r$ during each bit period can be expressed as: 


$$
r=s_{s i g}+\sum_{j=1}^{N-2} s_{\mathrm{j}}+\mathrm{w}_{\text {thermal }}
$$

Where ${ }^{s}$ sig is the signal from the transmitter, $s_{j}$ is the signal from an interfering node $\mathrm{j}$, and ${ }^{\mathrm{w}}$ thermal is the thermal noise signal. Next step is to derive these three components. The power of the intended signal from the transmitter as observed at the receiver can be written as [5]

$$
P_{r}=\frac{\alpha P_{t}}{r_{\text {link }}^{\gamma}}
$$

Where:

$$
\alpha \triangleq \frac{G_{t} G_{r} c^{2}}{(4 \pi)^{2} f_{c}^{2}}
$$

And $P_{t}$ is the transmit power, $G_{t}$ and $G_{r}$ are the transmitter and receiver antenna gains, $f_{c}$ is the carrier frequency, and $c$ is the speed of light. Here, it is assumed that the antennas at the nodes are omnidirectional $\left(\mathrm{G}_{\mathrm{t}}=\mathrm{G}_{\mathrm{r}}=1\right)$, and the carrier frequency is $2.4 \mathrm{GHz}$.

Fig. 3 illustrates a scenario where the receiver is at the center of the network and the other nodes are grouped in concentric square tiers. Consider a potential interfering node $\mathrm{j}$ at $\mathrm{a}$ distance $\mathrm{j} \mathrm{r}_{\text {link }}$ from the receiver, where $\mathrm{j}$ is a multiplicative factor depending on the position of node $\mathrm{j}$. The interference power from node $\mathrm{j}$ can be written as:

$$
P_{\text {int } j}=\frac{\alpha P_{\mathrm{t}}}{\left(v_{\mathrm{j}} r_{\text {link }}\right)^{\gamma}}=\frac{P_{\mathrm{r}}}{v_{\mathrm{j}}^{\gamma}}
$$

The probability that an interfering node will transmit and cause interference depends on the MAC protocol employed. Considering the RESGO MAC protocol and assuming that each node transmits packets with fixed length $\mathrm{L}$ (dimension: [b/pck]), it can be shown that the interference probability is equal to the probability where an interfering node transmits during a vulnerable interval of duration $\mathrm{L}=\mathrm{Rb}$ [17]. This probability can be written as:

$$
\begin{gathered}
p_{\text {tran }}=1-e^{-\frac{\lambda_{t} L}{R_{b}}} \\
s_{j}=\left\{\begin{array}{cc}
\sqrt{\frac{E_{b}}{v_{j}^{\gamma}}} & \text { with probability } \frac{1}{2} p_{\text {tran }} \\
-\sqrt{\frac{E_{b}}{v_{j}^{\gamma}}} & \text { with probability } \frac{1}{2} p_{\text {tran }} \\
0 & \text { with probability } 1-p_{\text {tran }}
\end{array}\right.
\end{gathered}
$$

Assuming that the threshold for bit detection is placed at 0 , the bit error probability can be written as:

$$
P\{\text { bit error }\}=B E R_{\text {link }}=\sum_{\overline{s_{\text {lnt }}}} P\left\{\sqrt{E_{b}}+\sum_{j=1}^{N-2} s_{j}+w_{\text {thermal }}<0 \mid \vec{s}_{\text {int }}\right\} P\left\{\vec{s}_{\text {int }}\right\}
$$

Where the summation is carried out over all possible interference configurations given by ${ }^{\mathrm{s}}$ int. Given the random

$$
P\left\{\sqrt{E_{b}}+\sum_{j=1}^{N-2} s_{j}+w_{\text {thermal }}<0 \mid \vec{s}_{i n t}\right\}=Q\left(\frac{\sqrt{E_{b}}+\sum_{j=1}^{N-2} s_{j}}{\sigma}\right),
$$

Where:

$$
\sigma=\sqrt{F k T_{0} / 2}
$$

The BER at the end of a route with $n_{\text {grid }}$ links can be denoted in the following equation:

$$
B E R_{\text {route }}^{\text {grid }}=1-\left(1-B E R_{\text {link }}\right)^{\bar{n}_{\text {grid }}}
$$

$$
I_{\text {grid }} \triangleq \frac{1}{r_{\text {link }}^{\gamma}} \sum_{i=1}^{i_{\max }}\left[\frac{4}{i^{\gamma}}+\frac{4}{(\sqrt{2} i)^{\gamma}}+\sum_{j=1}^{i-1} \frac{8}{\left(\sqrt{i^{2}+j^{2}}\right)^{\gamma}}-1\right]
$$

The first term in the summation corresponds to the four nodes in each tier that are aligned with the receiver at the center of the network. The second term in the summation corresponds to contribution from the four nodes at the corners of each tier at distance $\sqrt{2}$ ir_link from the receiver. Lastly, the last term in the summation corresponds to the contribution of all the other nodes in each tier. Note that each tier has eight nodes at distance $\sqrt{ }\left(i^{\wedge} 2+j^{\wedge} 2\right)$ rlink from the receiver, for $j<i$. With the RESGO MAC protocol, each node transmits with probability ptran, and thus:

The average interference power can be written as:
The average interference power as observed by the receiver. Since each node transmits with a common power $\mathrm{Pt}$, if all nodes simultaneously transmit, it can be shown that the total interference power experienced by the receiver at the center of the network is [17]

$$
P_{\text {total }}^{\text {grid }}=\alpha P_{t} I_{\text {grid }}
$$

Where:

$E\left[P_{\text {int }}^{\text {grid }}\right]=p_{\text {tran }} P_{\text {total }}^{\text {grid }}=\left(1-e^{-\frac{\lambda_{t} L}{R_{b}}}\right) P_{\text {total }}^{\text {grid }}$

Considering BPSK signaling, and assuming that the interfering noise is Gaussian, the BER on each link of a route can be written as [4]

$$
B E R_{\text {link }_{\text {grid }}}=Q\left(\sqrt{\frac{2 \alpha P_{t} / r_{\text {link }}^{\gamma}}{P_{\text {thermal }}+E\left[P_{\text {int }}^{\text {grid }}\right]}}\right)
$$


The route BER can then be evaluated using the previous equation.

A typical BERgrid can be observed in Fig. 5. This is intuitively expected because after the network becomes dense enough, increasing the node spatial density (i.e., reducing the hop length) no longer improves the link SNR, as the interfering nodes also become close enough to the receiver. Yet, the route BER predicted by the Gaussian assumption for the interference signal produces a much lower BER floor.

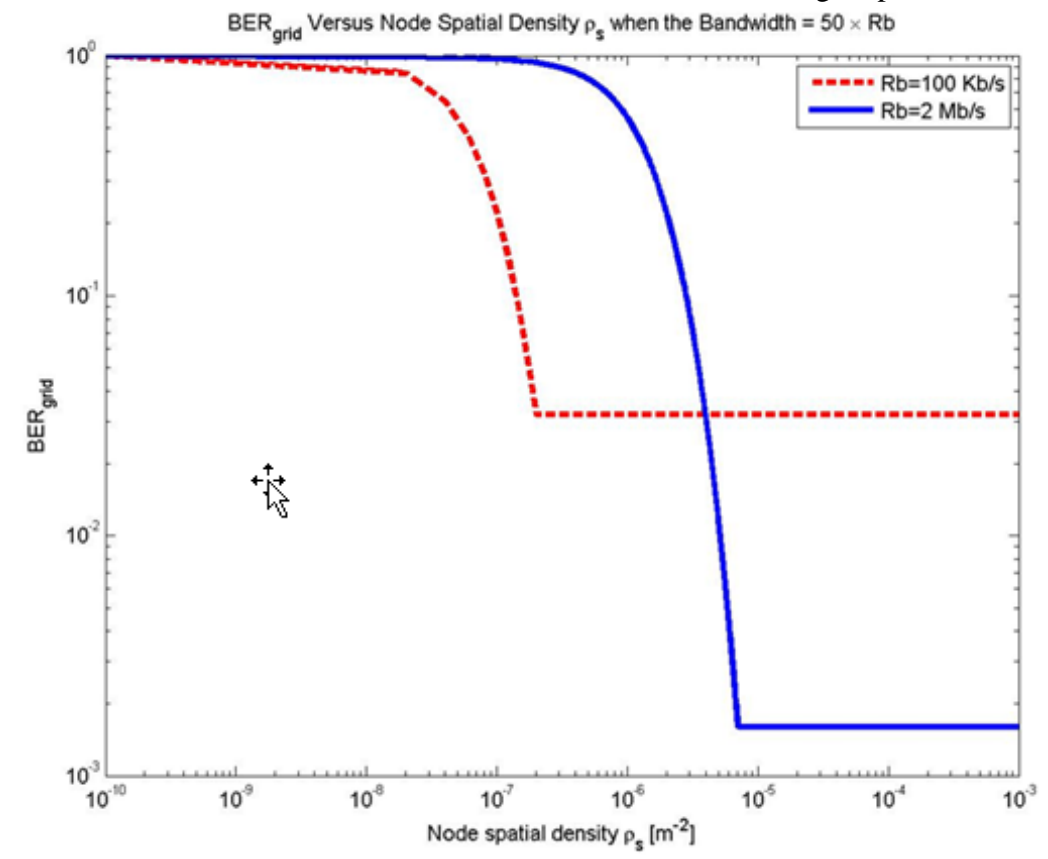

Fig 4: BERgrid versus node spatial density observed at specific data rate $\mathbf{R b}$

A typical BERgrid varies with Node spatial density when $\mathrm{Rb}=100 \mathrm{~Kb} / \mathrm{s}$ or $2 \mathrm{Mb} / \mathrm{s}$ can be observed in Figure 4 . This is intuitively expected because, after the network becomes dense enough, increasing the node spatial density (i.e., reducing the hop length) no longer improves the link SNR, as the interfering nodes also become close enough to the receiver.

However, the route BER predicted by the Gaussian assumption for the interference signal presents a much lower
BER floor. Via a careful analysis, the route BER floor can be approximated with the following expression:

$$
B E R_{\text {floor }} \approx \frac{3 \bar{n}_{\text {grid }} \lambda_{t} L}{4 R_{b}}
$$

Motivated by the above considerations, one finds out that an accurate approximate expression for the route BER can be written as:

$$
B E R_{\text {route }} \cong \max \left\{\left(1-\left[1-Q\left(\sqrt{\frac{2 \alpha P_{t} / r_{\text {link }}^{\gamma}}{P_{\text {thermal }}+E\left[P_{\text {int }}^{\text {grid }}\right]}}\right)\right]^{\bar{n}_{\text {grid }}}\right), \frac{3 \bar{n}_{\text {grid }} \lambda_{t} L}{4 R_{b}}\right\}
$$

\section{OPTIMAL COMMON TRANSMIT POWER}

In this section, the optimal common transmitted power for ad hoc wireless networks will be analyzed with grid topology.

\subsection{Optimal Common Transmitted Power for Networks}

With Square Grid Topology, the exact BER analyzed previously, one can always numerically find the minimum transmit power that would satisfy the desired BER threshold, provided that the BER threshold is above the BER floor. In this section, the optimal transmitted power for a given

$$
1-\left[1-Q\left(\sqrt{\frac{2 \alpha P_{t} / r_{\text {link }}^{\gamma}}{P_{\text {thermal }}+E\left[P_{\text {int }}^{\text {grid }}\right]}}\right)\right]^{\bar{n}_{\text {grid }}} \leq B E R_{\text {th }}
$$

Substituting FkT0Rb for Pthermal, using the previous given expression, one obtains the following expression for the optimal transmit power: maximum tolerable route BER from the approximate route BER expression as given before. In a network with grid topology, the common transmit power used by each node must be large enough so that the BER at the end of a multihop route with an average number of hops ngrid, as shown, is lower than the maximum tolerable value, denoted as BERth. If the required BERth is higher than BERfloor, a power that can satisfy this requirement exists. This transmitted power is such that the following inequality must be satisfied:

$$
1-\left(1-B E R_{\text {link }_{\text {grid }}}\right)^{\bar{n}_{\text {grid }}} \leq B E R_{\text {th }}
$$

It can be rewritten as:

$P_{t}^{*}=F k T_{0} R_{b}\left[\frac{2 \alpha}{r_{\text {link }}^{\gamma} \psi}-\alpha\left(1-e^{-\frac{\lambda_{t} L}{R_{b}}}\right) I_{\text {grid }}\right]^{-1}$

(dimension: [Watt]), where: 


$$
\begin{array}{r}
\psi \triangleq\left\{Q^{-1}\left[1-\left(1-B E R_{\text {th }}\right)^{\left.\frac{1}{\overline{\bar{n}}_{\text {grid }}}\right]}\right\}^{2}\right. \\
B E R_{\text {floor }} \leq B E R_{\text {th }}
\end{array}
$$

For a given data rate $\mathrm{Rb}$, node spatial density, number of nodes in the network, antenna gains, and carrier frequency (depends on them). It is difficult to find a closed-form expression for the optimal transmit power. In this case, the authors resort to simulations. For a given data rate and transmit power, the authors compute the average route BER with the approach described in the previous section. The same process is repeated for different combinations of data rate and transmits power. Finally, the authors obtain the average route BERs for different combinations of data rate and transmit power, and these are used to obtain contours of power-data rate pairs in correspondence to which the route BER is equal to maximum tolerable BERth values.

Network lifetime is an important performance indicator for wireless ad hoc and sensor networks. There are several definitions of network lifetime. While network lifetime is defined as the time to the first node failure in [5] and [6], it is defined in terms of the fraction of surviving nodes in the network in [7]. In this paper, following [5] and [6], the authors consider the time to the first node failure as the network lifetime (i.e., a worst case approach). This section presents a simple analysis to compute the average node lifetime. It is assumed that the RESGO MAC protocol is used, and that every node has an initial finite battery energy denoted by $E_{\text {batt }}$. For a given data rate $\mathrm{R}_{b}$, the time taken to transmit one packet is $\mathrm{L}=\mathrm{R}_{\mathrm{b}}$. Therefore, the total amount of energy consumed per transmitted packet can be written as:

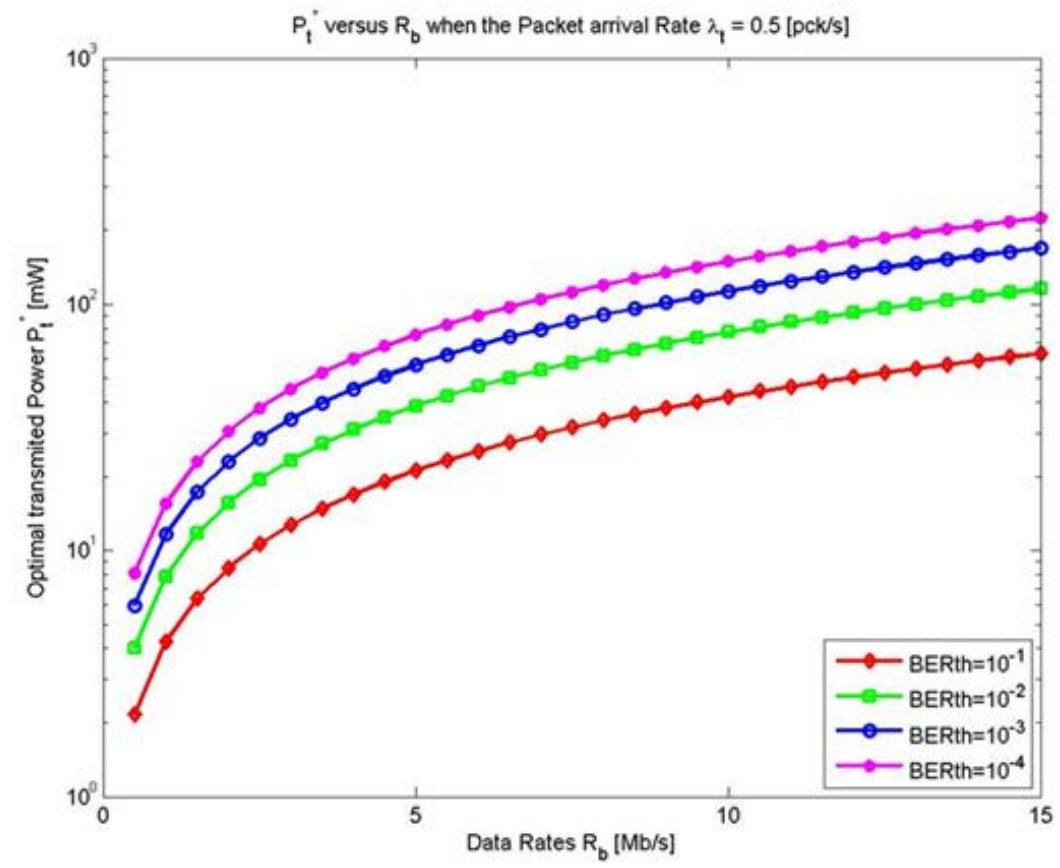

Fig 5: Optimal transmitted power $P_{-} t^{\wedge *}$ Versus data rate $R b$ when the arrival rate $\lambda t=0.5$ [pck.s]

$$
E_{\text {packet }}=P_{t} \times \frac{L}{R_{b}} \quad(\text { dimension: }[\text { Joules] }) .
$$

Since packets are transmitted with average rate $\lambda t$, the average energy depleted per second is simply $\mathrm{E}_{\text {packet }}$ Lastly, the total time it takes to completely exhaust the initial battery energy can be written as:

$$
\left.\tau=\frac{E_{\text {batt }}}{\lambda_{t} E_{\text {packet }}}=\frac{E_{\text {batt }} R_{b}}{\lambda_{t} L P_{t}} \quad \text { (dimension }:[\mathrm{sec}]\right)
$$

Note that due to the assumption of uniform traffic, on average, all nodes consume their batteries at the same time. Moreover, this simple analysis does not consider the energy consumed when a node is receiving and processing packets. In reality, the lifetime of a node is shorter than what this analysis predicted. Thus, transmitting is the most "expensive" activity (from a battery consumption perspective). Power consumption in idle and receiving modes can be considered by properly extending the proposed approach. Fig 5 shows the variation of the Optimal transmitted power $\mathrm{P}_{-} \mathrm{t}^{\wedge *}$ with the data rate $\mathrm{R}_{\mathrm{b}}$ when the arrival rate $\lambda t=0.5$ [pck.s]. From this analysis, the figure depicts that the optimal transmitted power increases when $R_{b}$ increases, but increases when the BERth is decreasing. Fig 6 represents the same analysis from a different view. It shows the variation of the Optimal transmitted power $\mathrm{P}_{-} \mathrm{t}^{\wedge *}$ with the arrival rate $\lambda \mathrm{t}$ when the BERth-0.01 analyzed with different data rate $\mathrm{Rb}$. The figure also shows clearly that the optimal power is increasing with $R_{b}$. Meanwhile, it is almost fixed with the arrival rate. 


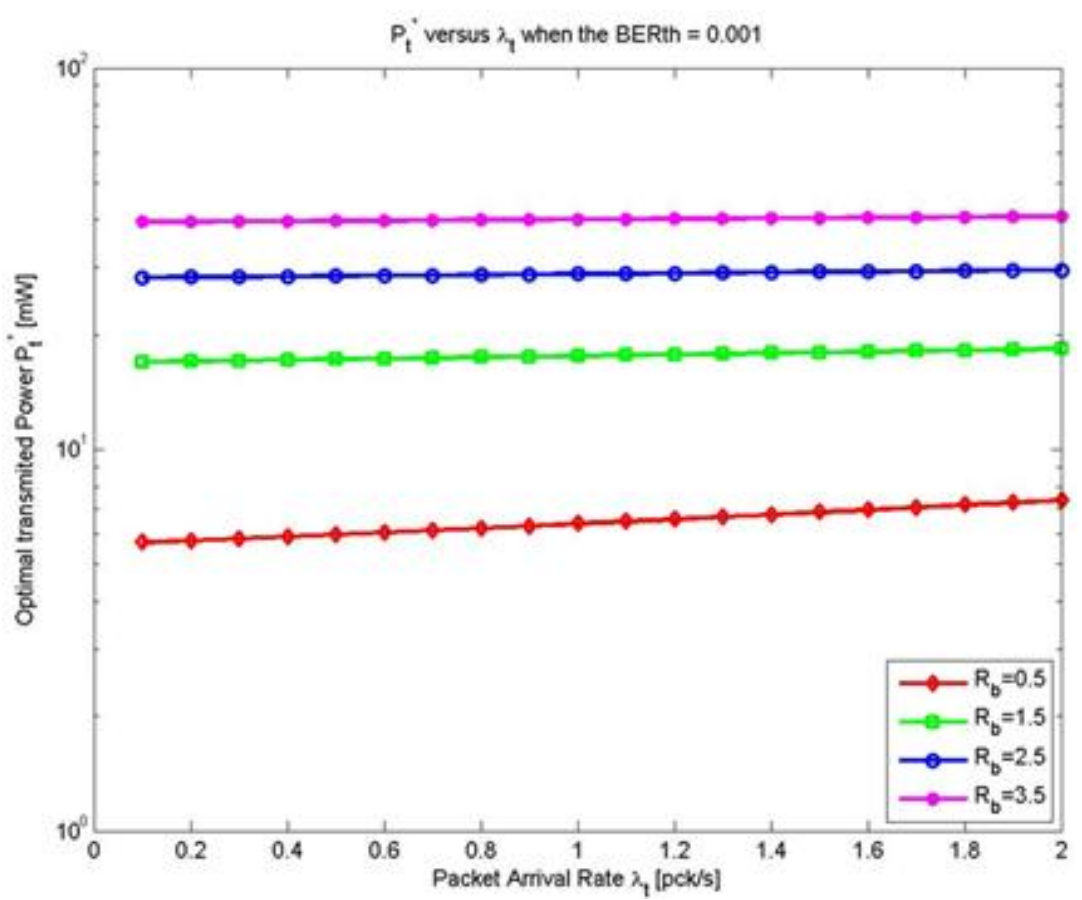

Fig 6: Optimal transmitted power $P_{-} t^{\wedge *}$ Versus the arrival rate $\lambda t$ when the BERth- 0.01 analyzed with different data rate $R b$

\section{CONCLUSION}

In this paper, a detailed investigation of the optimal common transmits power for wireless sensor networks are introduced. Particularly, the optimal common transmit power has been defined as the minimum transmit power sufficient to preserve network connectivity. This paper considered the connectivity from a viewpoint of considering the characteristics of a wireless communication channel. This study shows that, for a given data rate and a given maximum tolerable BER at the end of a multihop route, an optimal transmit power exists. Moreover, for a given value of the maximum tolerable route BER, a global optimal data rate exists for which the optimal common transmit power is the minimum possible. These shows that the data rate, if selected carefully, can grant significant savings in terms of transmit power, prolonging the devices' lifetime battery and network.

This work is useful in designing wireless sensor networks. Since the node spatial density of a sensor network is typically known in advance, a network designer can determine the optimal power and the optimal data rate to operate. On the other hand, when the transmitted power and the data rate of a sensor node is preconfigured, a network designer can use the analyzed approach to determine the minimum node spatial density required to keep the network connected. In other words, the common power control scheme performs well when each link in the network experiences similar propagation pathloss. When variation in propagation pathloss occurs, the transmit power needs to be increased substantially even though there is only one link per route which experiences high pathloss. A possible solution to this problem is to add intelligence into the routing protocol. More precisely, a routing protocol that takes link quality as one of its route selection criteria could be an attractive option. However, if it is not possible to avoid a high propagation loss link by means of routing, local power control may be required.

In addition, there are trade-offs between robustness to change in network connectivity and network longevity. Considering a transmit power higher than the optimal value helps preserve connectivity of networks in the presence of node spatial density change. However, this comes at the expense of node/network lifetime.

\section{REFERENCES}

[1] C. C. Tseng and K.-C. Chen, "Power Efficient Topology Control in Wireless Ad Hoc Networks," Proc. IEEE Wireless Comm. And Networking Conf. (WCNC), vol. 1, pp. 610-615, Mar. 2004.

[2] Chun-Hung Liu, Beiyu Rong, and Shuguang Cui, "Optimal Discrete Power Control in Poisson-Clustered Ad Hoc Networks", IEEE TRANSACTIONS ON WIRELESS COMMUNICATIONS, VOL. 14, NO. 1, JANUARY 2015.

[3] D. Sylvia, Jeevaa K. Atiravan, and D. Srinivasa Rao, "Implementation of Power Control in a Resource Constrained Wireless Ad-Hoc Network", Middle-East Journal of Scientific Research 23 (4): 756-760, 2015.

[4] G. Ferrari, S. Panichpapiboon, N. Wisitpongphan, R. Chokshi, and O.K. Tonguz, "Impact of Mobility on the BER Performance of Multi-Hop Ad Hoc Wireless Networks," Proc. IEEE Global Telecomm. Conf. (GLOBECOM), pp. 882-886, Nov. 2004.

[5] G. Ferrari, S.A. Malvassori, M. Bragalini, and O.K. Tonguz, "Physical Layer-Constrained Routing in AdHoc Wireless Networks: A Modified AODV Protocol with Power Control," Proc. Int'l Workshop Wireless AdHoc Networks (IWWAN '05), May 2005.

[6] J. Boyer, D. Falconer, and H. Yanikomeroglu, "Multihop Diversity in Wireless Relaying Channels," IEEE Trans. Comm., vol. 52, no. 10, pp. 1820-1830, Oct. 2004.

[7] J. Boyer, D.D. Falconer, and H. Yanikomeroglu, "On the Aggregate SNR of Amplified Relaying Channel," Proc. IEEE Global Telecomm. Conf. (GLOBECOM), vol. 5, pp. 3394-3398, Nov. 2004. 
[8] Junfeng Qiao, Sanyang Liu, Xiaogang Qi1 and Gengzhong Zheng, "TRANSMISSION POWER CONTROL IN WIRELESS SENSOR NETWORKS UNDER THE MINIMUM CONNECTED AVERAGE NODE DEGREE CONSTRAINT", INTERNATIONAL JOURNAL ON SMART SENSING AND INTELLIGENT SYSTEMS VOL. 8, NO. 1, MARCH 2015.

[9] O. K. Tonguz and G. Ferrari, Ad Hoc Wireless Networks: A Communication-Theoretic Perspective. Wiley, 2006.

[10] O. K. Tonguz and G. Ferrari, "Is the Number of Neighbors in Ad Hoc Wireless Networks a Good Indicator of Connectivity?" Proc. Int'l Zurich Seminar Comm. (IZS '04), pp. 40-43, Feb. 2004.

[11] S. Panichpapiboon, G. Ferrari, and O.K. Tonguz, "Optimal Common Transmit Power in Ad Hoc Wireless Networks," Proc. IEEE Int'l Performance, Computing, and Comm. Conf. (IPCCC), pp. 593-597, Apr. 2005.

[12] L. S. Vijaya Kumar, Ananda babu J, "A SURVEY OF TOPOLOGY CONTROL OF MULTIHOP WIRELESS NETWORKS FOR ENERGY EFFICIENCY AND ROUTING IN AD HOC NETWORKS", International Journal of Advanced Technology in Engineering and
Science www.ijates.com, Volume No 03, Special Issue No. 01, March 2015 ISSN (online): 2348 - 7550.

[13] N. Wisitpongphan, G. Ferrari, S. Panichpapiboon, J. Parikh, and O.K. Tonguz, "QoS Provisioning Using BER-Based Routing for Ad Hoc Wireless Networks," Proc. IEEE Vehicular Technology Conf. (VTC), May 2005.

[14] O. K. Tonguz and G. Ferrari, "A CommunicationTheoretic Approach to Ad Hoc Wireless Networking," Proc. 2006 Int'l Workshop Wireless Ad-Hoc Networks (IWWAN ’06), Invited Paper, June 2006.

[15] Sooksan Panichpapiboon, Gianluigi Ferrari, and Ozan K. Tonguz, "Optimal Transmit Power in Wireless Sensor Networks", IEEE TRANSACTIONS ON MOBILE COMPUTING, VOL. 5, NO. 10, OCTOBER 2006.

[16] S. Panichpapiboon, G. Ferrari, and O.K. Tonguz, "Sensor Networks with Random versus Uniform Topology: MAC and Interference Considerations," Proc. IEEE Vehicular Technology Conf. (VTC), pp. 2111-2115, May 2004.

[17] T. Elbatt and A. Ephremides, "Joint Scheduling and Power Control for Wireless Ad Hoc Networks," IEEE Trans. Wireless Comm., vol. 3, no. 1, pp. 74-85, Jan. 2004. 